\title{
Applying AHP Technique for Trust Evaluation in the Semantic Web
}

\author{
Bagher Rahimpour Cami and Amin Khodabandeh Amiri
}

\begin{abstract}
The increasing reliance on information gathered from the web and other internet technologies raises the issue of trust. Through the development of semantic Web,One major difficulty is that, by its very nature, the semantic web is a large, uncensored system to which anyone may contribute. This raises the question of how much credence to give each resource. We can't expect eachuser to know the trustworthiness of each resource, nor would we want to assign top-down or global credibility values due to the subjective nature of trust. Trust policiesand trust evaluation mechanisms are needed to filter untrustworthy resource. We tackle this problem by employing a trust model for evaluating trustworthiness ofeach resource. This proposed model uses semantic webmetadata, recommendation, and reputation as based factorfor evaluation algorithm. The weighting and Combination Methods are two main challenges for Proposed Trust evaluation algorithm. These factors have various type and semantic. Therefore we apply the AHP technique for trust evaluation that offers justification for trust decisions andcontrolled trust measurement.
\end{abstract}

Index Terms-AHP, content trust, hybrid trust model, semantic web, trust.

\section{INTRODUCTION}

With the expansion of the internet, users and services are often required to interact with unknown entities. Thus, one of the great challenges of the web is the problem of trust.

It is important for each user to identify trustworthy entities or correspondents with whom he/she should interact, and untrustworthy correspondents with whom he/she should avoid interaction [1].

Trust models have emerged as an important risk management mechanism in online communities [2]. The main goal of trust model is to detect of malicious or otherwise unreliable entities in a network [3].

A wide variety of literature now exists on trust evaluation, ranging from specific application to general models. Most prior approaches to trust models focus on entity centered issue such as credential and reputation that does not into account the content [4].

Credential -oriented trust model uses certificate authority to authentication and establish trust [2], [3], [5].

Reputation- oriented trust model uses experience or the experiences of others as recommendation, possibly combined to make trust decision about an entity [1], [6]-[8]. The next generation of the web is often characterized as the semantic web. In the semantic web, resources will no longer only be

Manuscript received September 9, 2011; revised December 7, 2012.

The authors are with Faculty of Computer \& IT Engineering, Mazandaran University of Science \& Technology, Babol, Iran (e-mail: rc_bagher@yahoo.com; aminkhodabandeh@gmail.com). intended for human consumers, but also for processing by machines, enabling intelligent information services, personalized web-site, and semantically empowered search-engines [9]. At the core of semantic web technologies lays RDF and languages and formalism based on it, most notably OWL [10]. Semantic web provides a new approach to trust model. Semantic web will enable us to judge on provided resources which one could call the content trust. Content trust is a trust judgment on a particular piece of information in a given context [3].

Our goal is to develop a general trust model that can be used for making rational decision in order to make optimal choice. The proposed trust model applies AHP [11] technique to build a suitable trust model that uses content and entity trust factors. The rest of the paper is recognized as follows:an overview of AHP technique is given in Section II, Section III explains trust evaluation process. Section IV explains the AHP technique weighting mechanism to hybrid trust components. In Section V, the experiment of proposed model is represented. At the end, the conclusion of paper is coming in Section VI.

\section{ANAlytical Hierarchy Procedure}

Submit your manuscript electronically for review. The analytical hierarchy procedure (AHP) is proposed by Saaty [11]. AHP was originally applied to uncertain decision problems with multiple criteria, and has been widely used in solving problems of ranking, selection, evaluation, optimization, and prediction decisions.

The AHP method is expressed by a unidirectional hierarchical relationship among decision levels. The top element of the hierarchy is the overall goal for the decision model. The hierarchy decomposes to a more specific criterion a level of manageable decision criteria is met [12]. Under each criterion, sub-criteria elements relative to the criterion can be constructed. The AHP separates complex decision problems into elements within a simplified hierarchical system [13].

The AHP usually consists of three stages of problem solving: decomposition, comparative judgment, and synthesis of priority. The decomposition stage aims at the construction of a hierarchical network to represent a decision problem, with the top level representing overall objectives and the lower levels representing criteria, sub-criteria and alternatives. With comparative judgments, expert users are requested to set up a comparison matrix at each hierarchy by comparing pairs of criteria or sub-criteria.

Finally, in the synthesis of priority stage, each comparison matrix is then solved by an eigenvector [17] method for determining the criteria importance and alternative 
performance. The purpose of the AHP enquiry in this paper is to construct a hierarchical evaluation system based on the resource attributes and entity reputation.

\section{Trust Evaluation Process}

The trust relation is created by a resource request issued by one entity to other entities of the network in order to fulfill a requisite .Trust relation[1] with respect to Fig. 1 includes 3 operators and labeled edge.

Entities in this relation are: Trustor, Trustee and Recommender Entities.

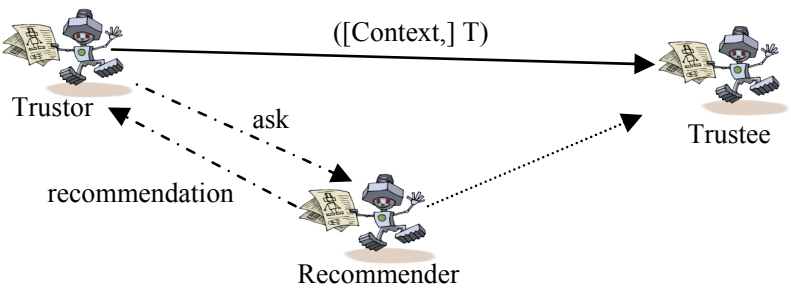

Fig. 1. Trust relation

Trustees are resource providers and claim to provide the requested resource for the trustor. The trust evaluation model should evaluate the trustworthiness of each trustee entity. In traditional web, the resources are represented as an undeductionable structures.

Therefore the trust evaluation is a separate process. In this structure of web, after gathering the claims of resource providers, the mechanism of trust evaluation is used. In this group of trust models, reputation of an entity is used to evaluate the trustworthiness [1], [7], [14]. With the emersion of semantic web, resources are defined and represented based on their constituent factors [4], [9], [15].

In this new theory toward the web structure, some factors are defined for each resource. These factors are defined by RDF, OWL languages and resource presentation is done with the help of XML syntax. [16].

With the growth and development of semantic web, an opportunity is provided where instead of human processing resources such as reading, browsing, form-filtering, intelligent services such as information filter, search agents and information broker can be provided [9]. In semantic web besides the syntactic form of resources, semantic content is also noted. Therefore the possibility of processing information contents by machine will be provided.

In the proposed trust evaluation model, the components of Hybrid Trust Model [3] are used. The fundamentaldifference resides in the way these factors are used. To use the components for evaluation trustworthiness of a resource provider, the AHP technique is used in this model.

These components include parameters of resource content [15] and the resource provider's reputation [9]. In this section, the extraction of the effective factors in trust evaluation of a resource which are used in AHP technique, takes place. Afterward, the factors which are effective and play an important role in trust evaluation of resources are investigated.

\section{Gathering Resource Providers as AHP ALTERNATIVES}

Trustee entities are used as AHP technique alternatives. Each of these entities is defined with an URL. Trustee entities are collected by web search engine as a result of processing a resource request [18].

Each entity represents one resource provider. In the proposed model, the trustworthiness of the resource provider and the submitted resource are both evaluated. Reputation and Content are used in evaluation of resource provider and submitted resource respectively. Trustee entities are using in the lowest Layer of AHP hierarchy as Alternatives.

\section{RETRIEVING RESOURCE ATtRIBUteS AS AHP CRITERIONS}

By offering resources in semantic web [18], the constituent Attributes of a resource can be retrieved by the semantic web search engines. In fact these constituent Attributes of a resource are retrieved in form of RDF Graphs [19], [9].

Each RDF Graph consists of the ternary object, Attribute, and value. The following sample, demonstrates a simple RDF Graph.

hasprice( http:// Book. Org/ISBN12515866, "\$62")

In the demonstrated relation, hasprice is an attribute, http://Book.Org/ISB12515866 is an object and "\$62" is considered to be a value. The semantic web search engines, gather each resource, in a form of ternary RDF graph. The retrieved Attributes are used as criterions for AHP technique. These attributes are used in investigating the priority of each alternative.

\section{Using Resource AtTRIBUtes to Establish CONTENT TRUST}

By retrieving resources with the semantic search engines [16], [18], [20] and extracting the resource attributes [9], [15] the AHP technique features will be provided.

In AHP technique, by retrieved features, the existing alternatives are pair compared according to these features. The criterions are used to pair comparison of each resource provider. With pair comparison; the pair matrix entries get filled.

In the AHP technique for carrying out the pair comparison of the alternatives according to the criterions, the experts and personal experiences are used. The experts fill pair matrixes according to their information and each criterion's value. Intelligent Agents can be used as are used for using experts. For each by requesting from the agents to any agents, their ideas about each criterion are retrieved from all of the providers.

Personal experiences are also used as an agent in this pair comparison. These expert agents gain necessary knowledge through a lot of interactions in a specific field.

\section{USING REPUTATION TO ESTABLISH ENTITY TRUST}

Reputation is the basis for many trust evaluation models [2], [21]. Reputation based models utilizes direct experience 
and other entity experiences called opinion recommender [1]. Because the trust evaluation in this method is based on the provider's behavior, it is also called entity trust.

In AHP for evaluating the trustworthiness of each entity, the pair comparison is used. For pair comparing the entities, recommender opinion and personal experiences are used as agents to construct pair matrix. By using AHP and performing operations on pair matrixes, the final weight vector for evaluating the trustworthiness of each provider entity is calculated.

\section{APPLyING AHP TECHNIQUE FOR TRUSt EVALUATION}

According to the Section 3, the process of trust evaluation can be represented as the Fig. 2. According to Fig. 2, $A_{1}$... $A_{2}$ are resource valued attributes and $E_{1} E_{m}$ is trustee entities. In this section, using of AHP technique to perform trust evaluation will be investigated.

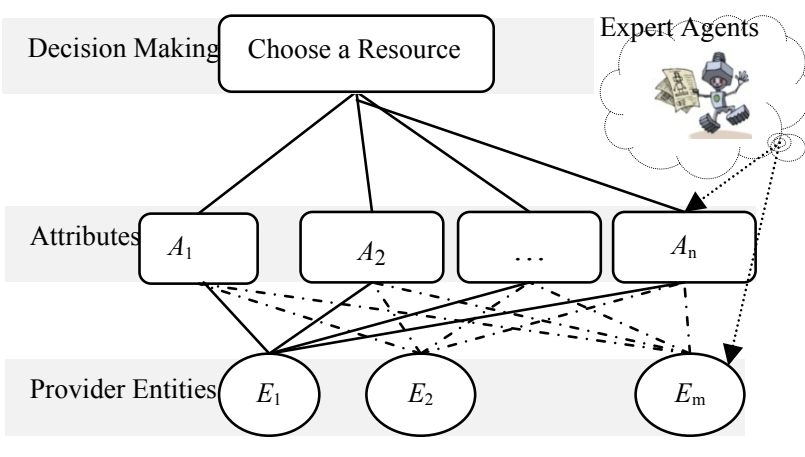

Fig. 2. AHP structure for trust evaluation

\section{A. Specifying Expert Agents for Weighting in AHP Algorithm}

To specify relative weight in AHP algorithm, Experts are needed in order to make the weighting matrix. In this model of trust evaluation, we use two types of Expert Agents:

Group1: Reputation-oriented Expert Agents: these Agents gain their knowledge through the direct experiences of the respective trustor entity in a period of time and then store it in its local database. These Agents use direct observations of trustor entities to decision-making; therefore after each interaction these Agents update their knowledge. They can use Statistical foundation for calculation [1], [7].

Group2: Recommender Expert Agent: we can use opinion of other Agents into weighting process. The opinions about the trustee can be gathered by acquiring information from Recommender Agents.

\section{B. Specifying Each Criterion's Priority}

Whenever a resource is provided, there are different criterions regarding the resource Attributes, each criterion's priority can be specified in choosing a resource.In order to specify the criterion's priority, pair comparing matrix is needed. Each entry of this pair comparing matrix denotes a weight ratio. This value calculates by performing a pair wise comparison of trustee based on each criteria from the prospect of an Expert agent. For specifying this value, the average of the agent's total scores is received. By performing AHP technique on the pair comparing matrix, weight of each criterion is extracted. That is shown in last column of Table I.

TABLE I: PAIR COMPARING MATRIX OF CRITERIONS

\begin{tabular}{|c|c|c|c|c|c|}
\cline { 2 - 6 } \multicolumn{1}{c|}{} & $\mathbf{A}_{\mathbf{1}}$ & $\mathbf{A}_{\mathbf{2}}$ & $\boldsymbol{\cdots}$ & $\mathbf{A}_{\mathbf{n}}$ & $\mathbf{W}$ \\
\hline $\mathrm{A}_{1}$ & 1 & $\mathrm{~b}_{12}$ & & & $\mathrm{w}_{1}$ \\
\hline $\mathrm{A}_{2}$ & $1 / \mathrm{b}_{12}$ & 1 & & & $\mathrm{~W}_{2}$ \\
\hline$\cdot$ & & & 1 & & $\cdot$ \\
\hline $\mathrm{A}_{\mathrm{n}}$ & & & & 1 & $\mathrm{~W}_{\mathrm{n}}$ \\
\hline
\end{tabular}

TABLE II: PAIR COMPARING MATRIX OF RESOURCE PROVIDERS

\begin{tabular}{|c|c|c|c|c|c|}
\hline $\mathbf{A}_{\mathbf{k} \in(\mathbf{1 . . n})}$ & $\mathbf{E}_{\mathbf{1}}$ & $\mathbf{E}_{\mathbf{2}}$ & $\ldots$ & $\mathbf{E m}$ & $\mathbf{W}$ \\
\hline $\mathbf{E}_{\mathbf{1}}$ & 1 & $\mathrm{a}_{12}$ & & & $\mathrm{~W}_{1}$ \\
\hline $\mathbf{E}_{\mathbf{2}}$ & $1 / \mathrm{a}_{12}$ & 1 & & & $\mathrm{~W}_{2}$ \\
\hline$\cdot$ & & & 1 & & $\cdot$ \\
\hline $\mathbf{E}_{\mathbf{m}}$ & & & & 1 & $\mathrm{~W}_{\mathrm{m}}$ \\
\hline
\end{tabular}

TABLE III: MATRIX OF TRUSTEE WEIGHTS RATIO FOR EACH CRITERION

\begin{tabular}{|c|c|c|c|c|}
\cline { 2 - 5 } \multicolumn{1}{c|}{} & $\mathbf{A}_{\mathbf{1}}$ & $\mathbf{A}_{\mathbf{2}}$ & $\ldots$ & $\mathbf{A}_{\mathbf{n}}$ \\
\hline $\mathbf{E}_{\mathbf{1}}$ & $\mathrm{w}_{11}$ & $\mathrm{w}_{12}$ & & $\mathrm{w}_{1 \mathrm{n}}$ \\
\hline $\mathbf{E}_{\mathbf{2}}$ & $\mathrm{w}_{21}$ & $\mathrm{w}_{22}$ & & $\mathrm{w}_{2 \mathrm{n}}$ \\
\hline$\cdot$ & $\cdot$ &. & & \\
\hline $\mathbf{E}_{\mathbf{m}}$ & $\mathrm{w}_{\mathrm{m} 1}$ & $\mathrm{w}_{\mathrm{m} 2}$ & & $\mathrm{w}_{\mathrm{mn}}$ \\
\hline
\end{tabular}

\section{Pair wise Comparison of Each Resource according to} the Specified Criterion

According to The AHP technique to calculate the weights ratio of each trustee entity, the following matrix is used. Expert Agent's opinion is used to perform pair wise comparison. The same matrix is used for other criterion. After calculating the above matrixes, the following matrix is formed. This matrix indicates the weights of each trustees ratio for each criterion.

\section{Calculating the Final Weight of Each Trustee}

After specifying weight for each criterion and pair comparing matrix of each resource based on the specified criterions, the final weight of each resource is calculated using the following formula:

$$
W_{E_{1}}=W_{A_{1}} * W_{11}+W_{A_{2}} \times W_{12}+\cdots+W_{A_{n}} \times W_{1 n}
$$

After calculating each resource weight, the resource's final priority is achieved and based on it, the resource with high priority can be selected.

\section{CONCLUSION}

We have presented a Hybrid Trust Model for evaluating trustworthiness in semantic web. Our scheme is based on AHP technique that it is applicable of Group decision-making. Considering trust a complex and multi-faceted thing, we use the AHP technique to capture content and reputation and recommendation to trust evaluation. The benefit of Hybrid trust model is that it provides a mechanism to apply all effective features in trust. This trust model is a new approach to trust evaluation and in next step we apply further semantic web feature.

\section{REFERENCES}

[1] J. Shi, G. Bochmann, and C. Adams, "A trust model with statistical foundation," IFIP vol. 173/2005, Springer, Toulouse, France, August 22-27, 2004, pp. 145-158. 
[2] D. Artz and Y. Gil, "A survey of trust in computer science and the Semantic Web," Web Semantics: Science, Services and Agents on the World Wide Web, vol. 5, no. 2, pp. 58-71, June 2007.

[3] B. Rahimpour and M. R. Matash borujerdi, "Using hybrid trust model for handling inaccurate resource," International Conference on Availability, Reliability and Security, CSS - ARES 2009-5-09-54.

[4] Y. Gil and D. Artz, "Towards content trust of web resources," Web Semantics: Science, Services and Agents on the World Wide Web 5, pp. 58-71, June 2007.

[5] M. Ma and C. Meinel. 'Independent Trust Intermediary Service," in Proc. of IADIS International Conf. Nov.2002.

[6] I. Ray and S. Chakraborty, "A vector model of trust for developing trustworthy systems," in European Symposium on Research in Computer Security, Springer-Verlag, pp. 260-275, 2004.

[7] I. Ray, S. Chakraborty, and U. Ray, "A trust management system based on a vector model of trust," University of Colorado State, Springer 2005.

[8] C. Ziegler and G. Lausen, "Propagation models for trust and distrust in social networks," Information Systems Frontiers, vol. 7, no. 4-5, pp. 337-358, Springer 2005.

[9] S. Decker, et al, "The semantic web - on the respective roles of XML and RDF," IEEE Internet Computing, 2000.

[10] V. D. Almendra and D. Schwabe, "Trust policies for semantic web repositories," in Proc. 5th International Semantic Web Conf. vol. 1, issue: 1 , pp. 5-9, 2006

[11] T. L. Saaty, The analytical hierarchy process, planning, priority resource allocation, RWS Publications, USA, 1980.
[12] L. M. Meade and A. Presley, "R\&D project selection using the analytic network process," IEEE Transaction on Engineering Management, vol. 49, no. 1, pp. 59-66, 2002.

[13] H. Fazlollahtabar and I. Mahdavi, "User/tutor optimal learning path in e-learning using comprehensive neuro-fuzzy approach," Elsevier, Educational Research Review, vol. 4, pp.142-155, 2009.

[14] J. Patel, W. T. L. Teacy, N. R. Jennings, and M. Luck. "A probabilistic trust model for handling inaccurate reputation resources," in Proc. 3th International Conf. Trust Management, 2005.

[15] G. Antoniou and F. V. Harmelen, "A Semantic Web Primer," 2004.

[16] B. Thuraisingham, "security standard for the web," Elsevier. Computer standards \& Interfaces, vol. 27, pp.257-268, 2005.

[17] G. Arfken, Mathematical methods for physicists, Orlando, FL: Academic Press, pp. 229-237, 1985

[18] V. S. Almendra and D. Schwabe, "Real-world trust policies," Semantic Web and Policy workshop, held in conjunction with international semantic web conference - ISWC' 05, 4., Galway, Ireland, 2005.

[19] J. C. Jeremy, C. Bizer, P. Hayes, and P. Stickler, "Named graphs, provenance and trust," in Proceedings of the 14th international conf. World Wide Web, ACM New York, NY, USA.

[20] R. Guha and R. McCool, "TAP: A semantic web platform," Computer Networks, vol. 42, issue 5, pp. 557-577, August 2003.

[21] G. C. Silaghi, A. E. Arenas, and L. M. Silva, "Reputation-based trust management systems and their applicability to grids," CoreGRID Technical Reports, TR-0064, 2007. 